\title{
An improved diffusion/compartmental model for transdermal drug delivery from a matrix-type device
}

\author{
Achim Göpferich and Geoffrey Lee \\ Institute for Pharmaceutical Technology and Biopharmaceutics, Heidelberg University, Heidelberg (Germany)
}

(Received 30 November 1990)

(Accepted 14 January 1991)

Key words: Transdermal drug delivery; Polymer matrix; Model

\begin{abstract}
Summary
A mathematical model is presented for the description of transdermal drug delivery from a matrix-type delivery device. The model is partly diffusional and partly compartmental in nature. The matrix and stratum corneum are both considered to be diffusion layers, connected to a three-compartment model representing the viable epidermis/dermis, plasma, and peripheral tissues. The diffusion equation was solved numerically for the two diffusion layers under non-sink conditions. The ordinary differential equations for the compartmental model were also solved numerically. Combination of the two numerical solutions yielded a model which directly relates the properties of the matrix to the profile of drug mass in the plasma and the urinary excretion profile. The model was first used to analyse data obtained from an in vivo trial of a matrix-type transdermal delivery device for the drug clenbuterol. Fitting of the model to the profile of drug concentration in the plasma, the urinary excretion profile, and the mass of drug remaining in the matrix with a modified simplex method yielded values for the model constants. These compared very favourably with independent values taken from the literature. Simulations of the influences of drug diffusivity within the stratum corneum, drug loading in the matrix, matrix thickness and drug diffusivity within the matrix on the profile of drug concentration in the plasma were then made. The model is not restricted to a steady state nor does it specify particular drug release kinetics from the matrix. It does assume isotropic diffusion layers and spontaneous partitioning at boundaries.
\end{abstract}

\section{Introduction}

Transdermal drug delivery can be viewed as a sequence of transport and distribution processes; the drug is first released from its delivery device and passes across the skin tissues into the systemic circulation, from where it is excreted via the kidneys into the urine (Guy and Hadgraft, 1980). The

Correspondence: G. Lee, Institut für Pharmazeutische Technologie und Biopharmazie, Im Neuenheimer Feld 366, D-6900 Heidelberg, Germany. resulting concentration profile in the plasma for drugs of short pharmacokinetic half-life is primarily rate-limited by transport across the outermost skin tissue, the stratum corneum. For drugs of long pharmacokinetic half-life the rate of excretion is a second rate-limiting factor (Tojo, 1988). These two rate-limiting processes cannot, however, be realistically tailored in a controlled manner to produce optimum drug concentration profiles in the plasma. It is much easier to adjust the rate of drug release from the delivery device, by altering, for the example of a polymer matrix-type system, the matrix thickness, drug loading or drug diffu- 
sivity within the matrix. Any model for transdermal drug delivery should, therefore, show directly how changes in these specific properties of the delivery device influence the drug concentration profile in the plasma.

None of the available, published models for transdermal drug delivery quite satisfies this objective. Compartmental models such as that illustrated in Fig. 1 (Guy et al., 1982), although capable of being combined with $\sqrt{t}$-drug release kinetics (Hadgraft, 1990), do not recognise the diffusional nature of drug passage through the skin. This inexactitude was overcome by Tojo (1988), who developed an elegant combined diffusion/compartmental model, also illustrated in Fig. 1. The non-steady-state diffusion of drug through the stratum corneum and viable epidermis is now recognised, and this transport process linked to a two-compartmental model representing distribution of the drug in the plasma and peripheral tissues. The specific properties of the delivery device are, however, still not considered. Indeed, the drug concentration at the outermost edge of the stratum corneum is assumed to be constant with this model, an assumption which is not justifiable and leads to simulated drug concentration profiles in the plasma that show a quite unrealistic steady-state phase. The model describes thus only the cases of pure skin- or pure elimination controlled transdermal drug delivery and effectively ignores the influence of the delivery device.

As part of a study of the transdermal delivery of the drug clenbuterol we have modified and extended the combined diffusion/compartmental approach to include non-steady-state diffusion within a matrix-type delivery device. We feel that the resulting model represents a more satisfying description of transdermal drug delivery than other models currently to be found in the literature. It shows directly how the drug concentration profile in the plasma during transdermal application can be influenced not only by the properties of the stratum corneum and underlying tissues but also by the specific properties of the matrix.

\section{Model for Transdermal Drug Delivery}

The non-steady-state model we propose is illustrated schematically in Fig. 2 and differs in three crucial points from that published by Tojo (1988). Firstly, both the matrix and stratum corneum are treated as diffusion layers. This is necessary by virtue of their high resistances to the diffusional transport of drug molecules. Both layers are taken to be isotropic, with drug diffusion characterised only by the diffusivities $D_{\mathrm{m}}$ and $D_{\mathrm{s}}$, respectively. We assume thereby that the drug molecules traverse a direct, linear pathway through the whole area of the stratum corneum. As such, the question of intra- versus intercellular pathways is not considered in this paper and the non-isotropic structure of the stratum corneum is ignored.

A spontaneous partitioning of drug at all boundaries is also assumed, since interfacial transfer is thought unlikely to be rate-determining for
Compartment Model

Diffusion/compartment Model
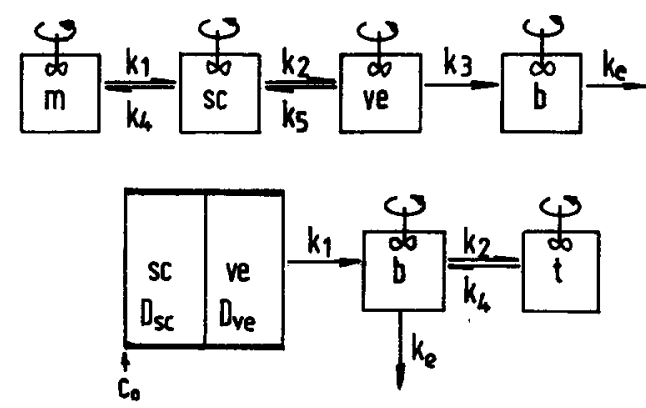

Guy et al.

(1982)

Tojo

(1988)

Fig. 1. Schematic representation of two literature models for transdermal drug delivery. (m) Delivery device; (sc) stratum corneum; (ve) viable epidermis; (b) plasma; ( $t$ ) peripheral tissues; $(D)$ diffusivity; $\left(k_{1}-k_{5}\right)$ microconstants; $\left(k_{\mathrm{e}}\right)$ elimination rate constant; $\left(c_{\mathrm{o}}\right)$ fixed drug concentration at outermost boundary of sc. 
percutaneous absorption (Albery and Hadgraft, 1979). The outer edge of the matrix $(x=-L)$ is insulated. If only linear diffusion with constant diffusivity occurs then the drug concentrations in the two layers, $c_{\mathrm{m}}(x, t)$ and $c_{\mathrm{s}}(x, t)$, can be described as functions of space, $x$, and time, $t$, by the one-dimensional form of Fick's Second Law (Crank, 1975):

$D \cdot c(x, t)_{x x}-c(x, t)_{t}=0$

where the subscripts define the respective partial derivatives. For completeness the model also specifies the existence of non-sink conditions at all boundaries.

We consider each of the underlying tissues of the viable epidermis/dermis, plasma and peripheral tissues to comprise a perfectly stirred compartment. In this we differ in a second point from Tojo (1988), who treated the viable epidermis as a separate diffusion layer. Although this tissue does comprise a distinct, morphological layer within the skin (Lee and Palicharla, 1986), drug diffusivi- ties within both viable epidermis and dermis are known to be approx. 1000 times greater than those within the stratum corneum (Scheuplein and Blank, 1971) and approx. 200-1000 times greater than those within the polymer matrix used for this study (Göpferich and Lee, 1991). Furthermore, the dermis is permeated extensively by capillaries, which extend up to the junction with the viable epidermis. Hence, our combination of the viable epidermis and dermis as a single compartment. Within each of the three compartments only the time-dependent drug mass, $m(t)$, need be defined. The first-order transfer of drug between the compartments is characterised in the usual fashion by microconstants. A third difference from Tojo (1988) is here the use of two microconstants $\left(k_{12}\right.$ and $k_{21}$ ) at the boundary between the viable epidermis/dermis compartment and the plasma. This allows the possible accumulation of drug external to this boundary, as caused, for example, by the retention of drug within the stratum corneum. Elimination from the plasma is characterised by $k_{\mathrm{e}}$. The change in drug mass with time within each
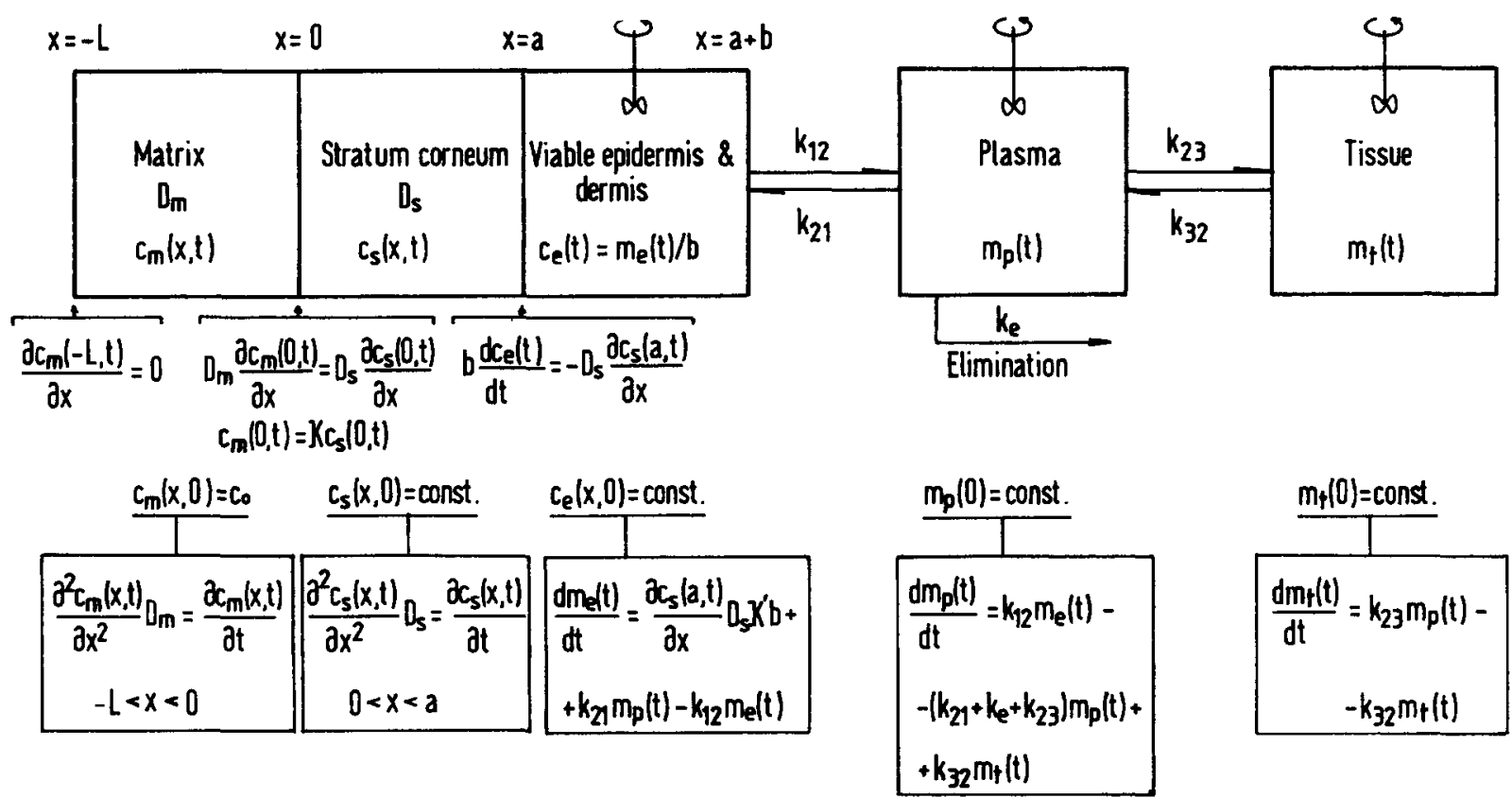

Fig. 2. Diffusion/compartmental model for transdermal drug delivery. $(x)$ space coordinate; $(t)$ time; $(c(x, t))$ drug concentration; $(m(t))$ drug mass; $(D)$ diffusivity; $\left(k_{12}, k_{21}, k_{23}, k_{32}\right)$ microconstants; $\left(k_{\mathrm{e}}\right)$ elimination rate constant; $\left(c_{0}\right)$ initial drug concentration in matrix. 
compartment, $\mathrm{d} m(t) / \mathrm{d} t$, can be described by an ordinary differential equation of first order, as shown in Fig. 2. The profile of drug concentration in the plasma, $c_{\mathrm{p}}(t)$, is now directly related to matrix thickness, $L$, drug loading, $c_{0}$, and drug diffusivity within matrix and stratum corneum.

\section{Methods}

An analytical solution to the model is difficult to obtain because the Laplace transform solution to the double-layer diffusional problem is not easy to invert (Göpferich and Lee, 1991) and the characteristic equation describing the three-compartment model with five microconstants is cubic and not directly solvable. A numerical solution can, however, readily be derived by using the CrankNicolson finite-difference method (Crank and Nicolson, 1947; Smith, 1987). The only complication involved occurs at the point $x=a$, where it is neccessary to change $c(x, t)$ into $m(t)$. The complete solution was programmed in Pascal on an Epson AX3 PC (80 386 processor with 80387 coprocessor).

We first used the model to analyse some in vivo data obtained during the transdermal application of the drug of interest to us - clenbuterol - from a polymer matrix. Rather than make a qualitative comparison we undertook a simultaneous leastsquares fit of the model to three sets of experimental data that had been obtained from a single patient wearing the device, viz. the profile of drug concentration in the plasma, $c_{\mathrm{p}}(t)$, the profile of mass of drug remaining in the matrix, $m_{\mathrm{m}}(t)$, and the profile of mass of drug excreted in the urine, $m_{\mathrm{u}}(t)$. An improved simplex method (Nelder and Mead, 1967) was used which yielded from the fit the best values for up to six of the model constants. Since the model has ten constants including the volume of distribution, $V_{\mathrm{d}}$, we decided to fix the values for $k_{23}, k_{32}$, and $k_{\mathrm{e}}$, these being available for clenbuterol in a published in vivo study (Zimmer, 1976). Furthermore, the partition coefficient at $x=a, K^{\prime}$, could reasonably be assumed to be unity. The best values for the remaining model constants $D_{\mathrm{m}}, K, D_{\mathrm{s}}, k_{12}, k_{21}$, and $V_{\mathrm{d}}$ were determined from the least-squares fit of the

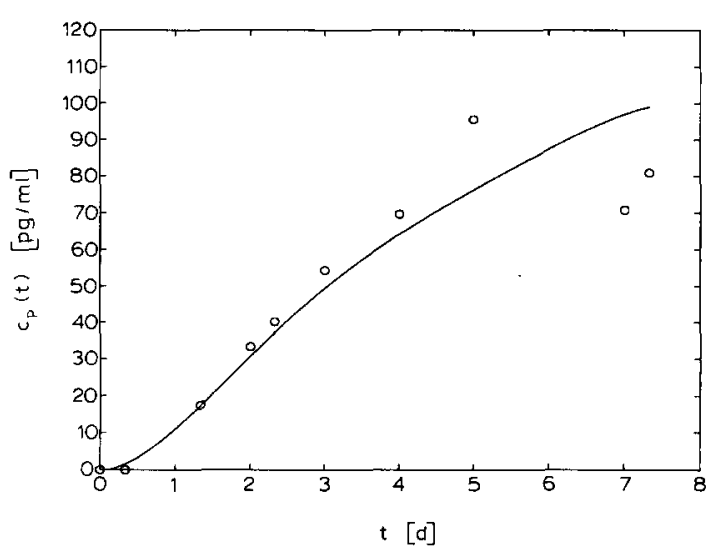

Fig. 3. Fitting of experimental data of drug concentration in plasma, $c_{\mathrm{p}}(t)$, [coordinates] obtained from an in vivo study of topical application of a clenbuterol-loaded polymer matrix to the diffusion/compartmental model [line] $\left(c_{\mathrm{o}}=1.25 \mathrm{mg} / 2 \mathrm{~cm}^{2}\right.$; $L=68 \mu \mathrm{m} ; K^{\prime}=1 ; a=15 \mu \mathrm{m} ; b=50 \mu \mathrm{m} ; k_{23}=0.2 \mathrm{~h}^{-1}$; $k_{32}=0.5 \mathrm{~h}^{-1} ; k_{\mathrm{e}}=0.028 \mathrm{~h}^{-1} ; A=2 \mathrm{~cm}^{2}$ ).

model to the three sets of independent, experimental data. The accuracy of the fit could then be judged by comparing the results obtained for the fitted constants with available literature values.

Once in possession of the model constants it was possible to produce simulated plasma profiles for clenbuterol. The influence of alteration in drug diffusivity within the stratum corneum, drug diffusivity within the matrix, drug loading of the matrix, and matrix thickness was simulated to yield profiles of drug concentration in the plasma versus time.

\section{Results and Discussion}

The coordinates shown in Fig. 3 are the experimentally determined values for $c_{\mathrm{p}}(t)$ obtained during in vivo application of a clenbuterol TTS to the inner arm. The curve represents the best leastsquares fit of the model to all data for $c_{\mathrm{p}}(t)$, $m_{\mathrm{m}}(t)$, and $m_{\mathrm{u}}(t)$. The accuracy of the fit can be judged by the values obtained for the model constants (Table 1), all of which are very good. The fitted value for $D_{\mathrm{m}}$, for example, agrees closely with a literature value obtained from measurement of drug release from the matrix in vitro (Göpferich and Lee, 1991). The value for $D_{\mathrm{s}}$ is somewhat 
TABLE 1

Values for the model constants together with equivalent literature values

\begin{tabular}{lcc}
\hline Model constant & $\begin{array}{c}\text { Value from } \\
\text { fit to model }\end{array}$ & $\begin{array}{l}\text { Value from } \\
\text { literature }\end{array}$ \\
\hline$D_{\mathrm{m}}\left(\mathrm{cm}^{2} \mathrm{~s}^{-1} \times 10^{11}\right)$ & 1.69 & $\begin{array}{c}2.14 \pm 0.63 \\
\left(c_{0}=6 \% \mathrm{w} / \mathrm{w}\right)^{\mathrm{a}}\end{array}$ \\
$D_{\mathrm{s}}\left(\mathrm{cm}^{2} \mathrm{~s}^{-1} \times 10^{12}\right)$ & 8.22 & $3.97 \pm 2.33^{\mathrm{a}}$ \\
$k_{12}\left(\mathrm{~h}^{-1}\right)$ & 1.47 & not available \\
$k_{21}\left(\mathrm{~h}^{-1}\right)$ & 12.6 & not available \\
$V_{\mathrm{d}}(\mathrm{l})$ & 113 & $85^{\mathrm{b}}$ \\
$K$ & 0.45 & not available \\
\hline
\end{tabular}

a From Göpferich and Lee (1991).

b From Zimmer (1976).

higher than that determined from measurements of drug permeation through human stratum corneum excised from the upper thigh (Göpferich and Lee, 1991). This difference can, however, readily be accounted for by variation in stratum corneum diffusivity and thickness between individuals and the two different body sites concerned, i.e. inner arm and thigh (Barry, 1983). It is at first glance surprising that the fitted value for $k_{21}$ is larger than that for $k_{12}$, as this indicates that the drug is accumulated somewhere within the system to the left of the point $x=(a+b)$. A closer scrutinisation of the in vivo data showed that this finding is correct; after 7 days, for example, only some $23 \%$ of the total mass of drug released from the matrix could be accounted for in the plasma, the peripheral tissue compartment, and the accumulated urine. This can most readily be explained by postulating the accumulation of a substantial amount of drug within the stratum corneum. Finally, the value obtained for $V_{\mathrm{d}}$ is also in close agreement with its literature value (Zimmer, 1976).

Our knowledge of the model microconstants now allows the effects of changes in these parameters on the plasma concentration profile to be simulated. Clenbuterol has a relatively long pharmacokinetic half-life of approx. $33 \mathrm{~h}$ (Zimmer, 1976). According to Tojo (1988) the plasma concentration profile of such a drug during transdermal delivery should be controlled mainly by this low rate of elimination. The simulations shown in
Fig. 4 illustrate, however, that the profile of drug concentration in the plasma, $c_{\mathrm{p}}(t)$, is still very sensitive to change in diffusivity within the stratum corneum, $D_{\mathrm{s}}$. The curves are simulated here over a realistic application period for a transdermal device of 7 days: each shows an initial lag time, reflecting non-steady-state diffusion through the stratum corneum. Compartmental models fail to illustrate this effect exactly (Guy et al., 1982), since they are perforce steady-state in nature. Noteably, none of the curves reaches a 'plateau' during the 7 days. This can only be observed by extending the simulation time substantially. Thus, Fig. 5 shows a simulation up to 56 days based on the results obtained from the fitting of the in vivo data. This simulated profile reaches a maximum $\left(t_{\max }\right)$, after which it declines, reflecting thereby the continual decrease in rate of drug release out of the polymer matrix with time. The error associated with an unrealistic steady-state phase as seen with Tojo's model (1988) is, therefore, avoided. The $t_{\max }$ of approx. 21 days seen here for clenbuterol is much longer than that found for clonidine during transdermal application in vivo (approx. 2 days; Arndts and Arndts, 1984). This arises from the much smaller stratum corneum diffusivity for clenbuterol compared to clonidine (the latter, $2 \times 10^{-10} \mathrm{~cm}^{2} \mathrm{~s}^{-1}$ according to Tojo, 1988) and the likely accumulation of clenbuterol

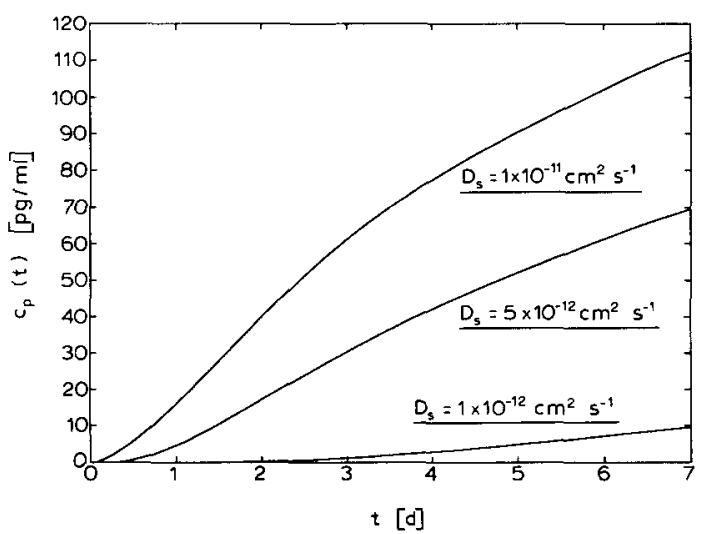

Fig. 4. Simulation of the influence of drug diffusivity in stratum corneum $\left(D_{\mathrm{s}}\right)$ on drug concentration in plasma, $c_{\mathrm{p}}(t)$ $\left(D_{\mathrm{m}}=4 \times 10^{-11} \mathrm{~cm}^{2} \mathrm{~s}^{-1} ; c_{0}=6 \% \mathrm{w} / \mathrm{w} ; K=1 ; K^{\prime}=1 ; L=\right.$ $200 \mu \mathrm{m} ; a=10 \mu \mathrm{m} ; b=50 \mu \mathrm{m} ; k_{12}=1.47 \mathrm{~h}^{-1} ; k_{21}=12.6$ $\mathrm{h}^{-1} ; k_{23}=0.2 \mathrm{~h}^{-1} ; k_{32}=0.5 \mathrm{~h}^{-1} ; k_{\mathrm{e}}=0.028 \mathrm{~h}^{-1}$ ). 


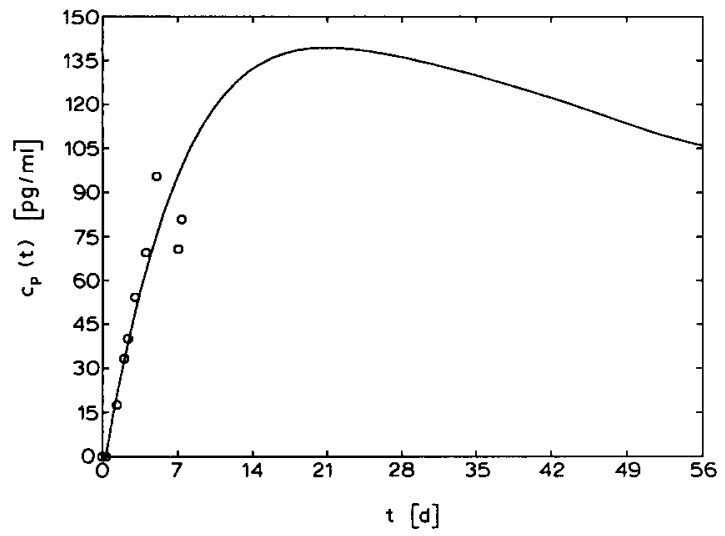

Fig. 5. Extended simulation for transdermal delivery of clenbuterol based on in vivo fit $\left(D_{\mathrm{m}}=1.69 \times 10^{-11} \mathrm{~cm}^{2} \mathrm{~s}^{-1} ; c_{0}=\right.$ $1.25 \mathrm{mg} / 2 \mathrm{~cm}^{2} ; K=1 ; D_{\mathrm{s}}=8.22 \times 10^{-12} \mathrm{~cm}^{2} \mathrm{~s}^{-1} ; K^{\prime}=1 ;$ $L=68 \mu \mathrm{m} ; a=15 \mu \mathrm{m} ; b=50 \mu \mathrm{m} ; k_{12}=1.47 \mathrm{~h}^{-1} ; k_{21}=12.6$ $\mathrm{h}^{-1} ; k_{23}=0.2 \mathrm{~h}^{-1} ; k_{32}=0.5 \mathrm{~h}^{-1} ; k_{\mathrm{e}}=0.028 \mathrm{~h}^{-1} ; A=2$ $\mathrm{cm}^{2}$ ).

within the epidermis as indicated by the current study.

The dependency of $c_{\mathrm{p}}(t)$ on $D_{\mathrm{s}}$ illustrates one possible source for the fluctuations often observed in drug plasma levels during transdermal administration. With the drug in question diffusivities in the range of $1.2-6.5 \times 10^{-12} \mathrm{~cm}^{2} \mathrm{~s}^{-1}$ have been reported for stratum corneum excised from the upper thigh of a single cadaver (Göpferich and Lee, 1991). This scatter is taken to arise from the natural variation in the non-isotropic composition of the stratum corneum. It is clear from Fig. 4 that variation in $D_{\mathrm{s}}$ within this range would lead to substantially differing $c_{\mathrm{p}}(t)$ profiles.

The effects of drug diffusivity within the matrix, $D_{\mathrm{m}}$ (Fig. 6a), are much less spectacular than those observed for $D_{\mathrm{s}}$. It is clear that alteration of this property of the matrix to manipulate transdermal drug delivery is limited in scope. The addition of plasticiser to the polymer matrix used here can, for example, readily increase $D_{\mathrm{m}}$ for clenbuterol by an order of magnitude from $2 \times$ $10^{-12}$ to $2 \times 10^{-11} \mathrm{~cm}^{2} \mathrm{~s}^{-1}$. This would have, however, only a small effect on $c_{\mathrm{p}}(t)$. The simulated profiles shown in Fig. $6 \mathrm{~b}$ illustrate clearly the linear dependence of $c_{\mathrm{p}}(t)$ on drug loading in the matrix. This property of the matrix has, therefore, a stronger influence on $c_{\mathrm{p}}(t)$ than does $D_{\mathrm{m}}$, but is, of course, limited by the solubility of the drug in the matrix ( $\sim 10 \% \mathrm{w} / \mathrm{w}$ for clenbuterol). The thickness of the matrix (Fig. 6c) has negligible influence on $c_{\mathrm{p}}(t)$, in agreement with previously published findings (Guy and Hadgraft, 1980).

\section{Acknowledgments}

We are most grateful to Heidelberg University (especially Herr Oberamtsrat Rainer Wolf) for generously providing funds for the purchase of an 80386 personal computer. Thanks are due to Boehringer Ingelheim $\mathrm{Kg}$ for its support of this project and for allowing us to use the in vivo data for the clenbuterol transdermal system.
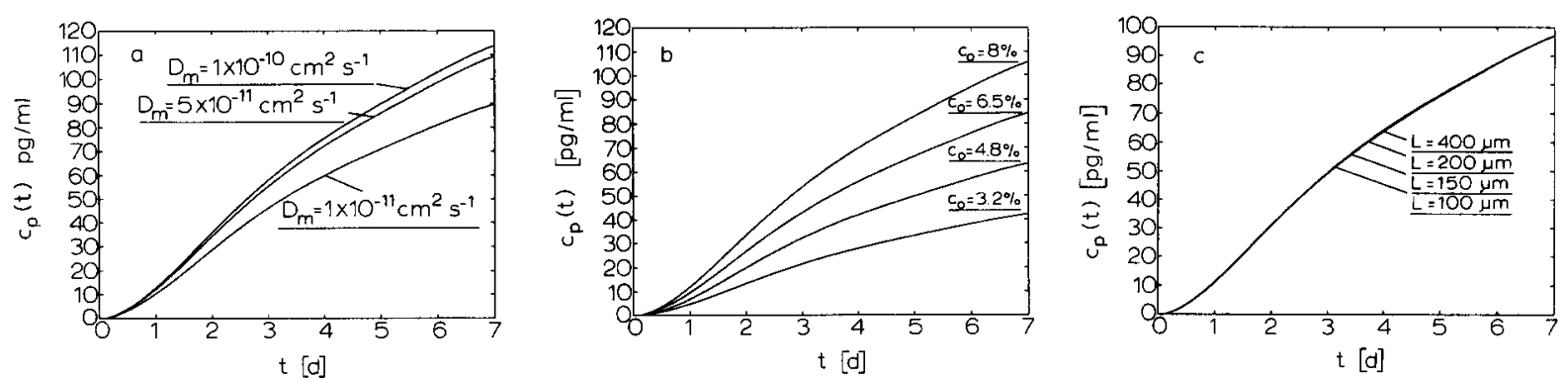

Fig. 6. Simulations of the influence of matrix properties on ' $c_{\mathrm{p}}(t)\left(K=1 ; K^{\prime}=1 ; a=10 \mu \mathrm{m} ; b=50 \mu \mathrm{m} ; k_{12}=1.47 \mathrm{~h}^{-1} ; k_{21}=12.6\right.$ $\left.\mathrm{h}^{-1} ; k_{23}=0.2 \mathrm{~h}^{-1} ; k_{32}=0.5 \mathrm{~h}^{-1} ; k_{\mathrm{e}}=0.028 \mathrm{~h}^{-1}\right)$. (a) Influence of drug diffusivity in matrix, $D_{\mathrm{m}}\left(D_{\mathrm{s}}=2 \times 10^{-12} \mathrm{~cm}^{2} \mathrm{~s}^{-1}\right.$; $\left.c_{0}=6 \% \mathrm{w} / \mathrm{w} ; L=200 \mu \mathrm{m}\right)$. (b) Influence of drug loading, $c_{0}\left(D_{\mathrm{m}}=4 \times 10^{-11} \mathrm{~cm}^{2} \mathrm{~s}^{-1} ; D_{\mathrm{s}}=2 \times 10^{-12} \mathrm{~cm}^{2} \mathrm{~s}^{-1} ; L=200 \mu \mathrm{m}\right)$. (c) Influence of matrix thickness, $L\left(D_{\mathrm{m}}=4 \times 10^{-11} \mathrm{~cm}^{2} \mathrm{~s}^{-1} ; D_{\mathrm{s}}=2 \times 10^{-12} \mathrm{~cm}^{2} \mathrm{~s}^{-1} ; c_{0}=6 \% \mathrm{w} / \mathrm{w}\right)$. 


\section{References}

Albery, W. and Hadgraft, J., Percutaneous absorption: interfacial transfer kinetics. J. Pharm. Pharmacol, 31 (1979) 6568.

Arndts, D. and Arndts, K., Pharmacokinetics and pharmacodynamics of transdermally administered clonidine. Eur. J. Clin. Pharmacol., 26 (1984) 79-85.

Barry, B., Dermatological Formulations, Dekker, New York, 1983, p. 133.

Crank, J., The Mathematics of Diffusion, 2nd. Edn, Oxford University Press, London, 1975, p. 15.

Crank, J. and Nicolson, P., A practical method for numerical evaluation of solutions to partial differential equations of the heat conduction type. Proc. Camb. Phil. Soc., 43 (1947) 50-67.

Göpferich, A. and Lee, G., Measurement of drug diffusivities in stratum corneum membranes and a polyacrylate matrix. Int. J. Pharm., (1991) in press.

Guy, R. and Hadgraft, J., A theoretical description relating skin permeation to the thickness of the applied medicament. Int. J. Pharm., 6 (1980) 321-332.
Guy, R., Hadgraft, J. and Maibach, H., A pharmacokinetic model for percutaneous absorption. Int. J. Pharm., 11 (1982) 119-129.

Hadgraft, J., Mathematical models of skin absorption. In Scott, R., Guy, R. and Hadgraft, J. (Eds), Prediction of Percutaneous Penetration, IBC Technical Services, London, 1990, pp.252-262.

Lee, G. and Palicharla, P., An examination of excised skin tissues used for in vitro membrane permeation studies. Pharm. Res., 3 (1986) 356-359.

Nelder J. and Mead, R., A simplex method for function minimisation. Comput. J., 10 (1967) 308-313.

Scheuplein, R. and Blank, I., Permeability of the skin. Physiol. Rev., 51 (1971) 702-747.

Smith, G., Numerical Solution of Partial Differential Equations: Finite Difference Methods, 2nd Edn, Oxford University Press, London, 1987, pp. 19-38.

Tojo, K., Concentration profile in plasma after transdermal drug delivery. Int. J. Pharm., 43 (1988) 201-205.

Zimmer, A., Einmalapplikation, Mehrfachapplikation und Metabolitenmuster von Clenbuterol beim Menschen. Arzneim.-Forsch. (Drug Res.), 26 (1976) 1446-1450. 